American Journal of Animal and Veterinary Sciences 3(1): 1-6, 2008

ISSN 1557-4555

(C) 2008 Science Publications

\title{
Rat Cardiomyocytes Express a Classical Epithelial Beta-Defensin
}

\author{
${ }^{1}$ Annika Linde, ${ }^{2}$ Gerald H. Lushington, ${ }^{1}$ Frank Blecha and ${ }^{3}$ Tonatiuh Melgarejo \\ Dept ${ }^{1}$ Anatomy \& Physiology, ${ }^{3}$ Human Nutrition, Kansas State University, Manhattan, KS 66506 \\ ${ }^{2}$ Molecular Graphics and Modeling Laboratory, University of Kansas, Lawrence, KS 66045
}

\begin{abstract}
Beta-defensins (BDs) are classical epithelial antimicrobial peptides of immediate importance in innate host defense. Since recent studies have suggested that certain BDs are also expressed in non-traditional tissues, including whole heart homogenate, and because effector molecules of innate immunity and inflammation can influence the development of certain cardiovascular disease processes, we hypothesized that BDs are produced by cardiomyocytes as a local measure of cardioprotection against danger signals. Here we report that at least one rat betadefensin, rBD1, is expressed constitutively in cardiomyocytes specifically isolated using positionablation-laser-microdissection (P.A.L.M. Microlaser Technologies). RT-PCR analysis showed expression of a single $318 \mathrm{bp}$ transcript in adult rat heart (laser-excised cardiomyocytes) and H9c2 cells (neonatal rat heart myoblasts). Moreover, the full length cDNA of rBD1 was established, and translated into a putative peptide with 69 amino acid residues. The predicted amino acid sequence of the adult rat cardiac BD-1 peptide displayed $99 \%$ identity with the previously reported renal $\mathrm{rBD} 1$, and $88 \%, 53 \%, 53 \%$ and 50\% identity with mouse, human, gorilla, and rhesus monkey BD1 respectively. Furthermore, structural analysis of the cardiac rBD1 showed the classical sixcysteine conserved motif of the BD family with an alpha-helix and three beta-sheets. Additionally, rBD1 displayed a significantly greater number of amphoteric residues than any of the human analogs, indicating a strong $\mathrm{pH}$ functional dependence in the rat. We suggest that $\mathrm{rBD} 1$, which was initially believed to be a specific epithelium-derived peptide, may be also involved in local cardiac innate immune defense mechanisms.
\end{abstract}

Keywords: Innate immunity, host defense peptides, heart, microdissection

\section{INTRODUCTION}

Defensins are master-players in innate immunity and one of the best characterized families of antimicrobial/host defense peptides identified in numerous species across the animal and plant kingdoms. To date, three categories of the defensin-family have been described in mammals, varying with respect to expression pattern and structural placement of six conserved cysteine residues ${ }^{[1]}$. Dissimilar to $\alpha$ defensins - which are principally produced by circulatory neutrophils and Paneth cells in the gut - $\beta$ defensins (BDs) are classical epithelium-derived peptides, whereas $\theta$-defensins thus far have been reported in non-human primates only ('mini defensins'). More recently, BDs have been identified in nonepithelial cell types also, including circulatory polymorph nuclear cells and tissues macrophages ${ }^{[1-3]}$. Defensins are commonly referred to as natural endogenous antibiotics. Still, these important molecules seem to function far beyond that of simple antimicrobial peptides, including features such as immunomodulatory and anti-tumor activities. Their expression can be either constitutive or inducible - classically instigated via Tolllike receptor mediated NF- $\mathrm{KB}$ (nuclear factor kappa-B) signaling secondary to pathogen associated molecular pattern recognition ${ }^{[3 ; 4]}$.

Cardiomyocytes have not traditionally been considered significant role-players in the orchestration of an innate immune response. Inflammation and innate immunity have, however, proven to be of major importance in a number of different cardiovascular disease processes, including atherosclerosis and myocarditis ${ }^{[5-9]}$. The Danger-Model of Immunity furthermore defines damage - more than mere foreignness - as the actual trigger of an innate immune response ${ }^{[10]}$. Moreover evidence exist that various sentinel cell types, including endotheliocytes, cardiomyocytes, fibroblasts and mast cells, can partake actively in an immune response along side more classical immune cells ${ }^{[9]}$. Local effector molecules of an innate immune response (incl. cardiac BDs) may thus

Corresponding Author: $\quad$ Tonatiuh Melgarejo, DVM MS PhD, Department of Human Nutrition, 143B Justin Hall 1

Kansas State University, Manhattan, KS 66506-1407 
be central for cardioprotection in infectious as well as non-infectious disease processes. Recent studies have incidentally found concomitant mRNA expression of select beta-defensins in whole heart homogenate from different species, including dBD1 in mice, eBD1 in horses, pBD1 in pigs, and hBD3 in humans ${ }^{[11-14]}$. Rat beta-defensin-1 (rBD1) mRNA expression has in addition been reported in a number of different organs, including the heart, based on screening of an array of whole tissue homogenates in rats with diabetic nephropathy ${ }^{[15]}$.

Similar to other organs, the heart contains a number of different cell types. Even though BDs have been detected in heart tissue, no studies have conclusively documented that the cardiomyocytes are in fact the actual source of the BD expression. We thus surmised that the rBD1 expression in whole heart homogenate can be ascribed directly to cardiac myocytes, and also that this can be identified in different life-stages. The presence of these key effector molecules of innate immunity may prove central to the heart's protective mechanisms towards an array of incoming dangers. This study provides evidence that laser-excised cardiomyocytes constitutively express at least one BD isoform.

\section{MATERIALS AND METHODS}

Whole heart tissue preparation: Whole hearts were excised from adult Wistar rats immediately after euthanasia, snap-frozen in liquid nitrogen and stored at $86^{\circ} \mathrm{C}$. All animal care and usage were in accordance with the Kansas State University IACUC (Institutional Animal Care and Usage) guidelines. The cryopreserved hearts were transferred individually to a LEICA CM3050 S Cryostat, and $1 / 2 \mathrm{~cm} \mathrm{x} 1 / 2 \mathrm{~cm}$ tissue blocks of the frozen specimens were excised at $-20^{\circ} \mathrm{C}$. Each tissue block was covered with TBS ${ }^{\mathrm{TM}}$ Tissue Freezing Medium (Triangle Biomedical Sciences, Durham, NC), sectioned at $10 \mu \mathrm{m}$, and transferred to $\mathrm{PALM}^{\circledR}$ MembraneSlides (Cat.No.1440-1000; P.A.L.M. Microlaser Technologies AG, Bernried, Germany) for a total number of 8-10 sections per slide. Tissue sections were fixed in $100 \%$ ethanol for $3 \mathrm{~min}$ at $-20^{\circ} \mathrm{C}$ immediately prior to staining. $\mathrm{H} \& \mathrm{E}$ (hematoxylin and eosin) staining was performed using a modified H\&E technique (IHC World) with all staining reagents kept on ice throughout the entire procedure.

H9c2 cell culture: A rat embryonic cardiomyocyte cell line $\mathrm{H} 9 \mathrm{c} 2$ was purchased through American type culture collection (ATCC, Cat. No. CRL-1446). Cells were cultured in DMEM (Invitrogen, Carlsbad, CA) supplemented with $10 \%$ fetal bovine serum, $100 \mathrm{U} / \mathrm{ml}$ penicillin, and $100 \mu \mathrm{g} / \mathrm{ml}$ streptomycin and grown up to $75-80 \%$ confluence before being used for experiments.

Position-Ablation-Laser Microdissection (P.A.L.M. Technology): The P.A.L.M. technology was used to selectively excise rat cardiomyocytes for further use in down-stream applications as described below. After $\mathrm{H}$ \& E staining on ice, each P.A.L.M. MembraneSlide was transferred individually to the P.A.L.M. ${ }^{\circledR}$ MicroBeam System (P.A.L.M. Microlaser Technologies AG, Bernried, Germany) for micro-dissection and pressure catapulting. Micro-dissection was used to collect cell clusters containing an average of 100 cardiomyocytes exclusively by catapulting cells into a collecting cap above the slide. To preserve RNA upon catapulting of selected cells the collecting cap was preloaded with $40 \mu \mathrm{l}$ of RLT buffer (Cat.No. 79216; QIAGEN, Valencia, CA). Once approximately 2,000 cells were catapulted, the cap was placed back into its original micro-vial and stored on ice for RNA extraction and further analysis.

RNA Extraction: RNA from laser-excised cardiomyocytes as well as $\mathrm{H} 9 \mathrm{c} 2$ cells (75\% confluency) was isolated using the RNeasy® Micro Kit 50 (Cat.No. 74004; QIAGEN, Valencia, CA) following manufacturer's directions. RNA yield and integrity were assessed using a NanoDrop Spectrophotometer (NanoDrop, Wilmington, DE) and Agilent 2100 Bioanalyzer (Agilent Technologies, Palo Alto, CA) respectively.

Identification and cloning of rBD1: To begin our identification of rat heart BD1, specific primers were designed using the cDNA sequence of the previously reported rat kidney $\beta$-defensin (GenBank No. AF093536): forward, 5'-TCT CTg CAC TCT ggA CCC TgA CTT-3' and reverse, 5'-CAA ACC ACT gTC AAC TCC TgC AAC-3'. Using the designed primers and total RNA (from the fetal and laser-excised cardiomyocytes) as template, a one-step RT-PCR reaction was performed using $2 \mu \mathrm{g}$ of total RNA purified from the cultured cells and micro-dissected cardiomyocytes respectively (OneStep RT-PCR Kit (25) Cat.No. 210210; QIAGEN, Valencia, CA). After electrophoresis of the PCR products on an agarose gel, a single band corresponding to approximately $300 \mathrm{bp}$ was excised from the gel and the DNA isolated and cloned using the TA Cloning ${ }^{\circledR}$ Kit (Cat.No. K2000-01 Invitrogen, Carlsbad, CA) and sequenced with an ABI 3700 DNA Analyzer at the K-State Sequencing and Genotyping Facility (Manhattan, KS). The correct 
sequence for the cloned product was verified by nucleotide sequencing. The efficiency of the RT procedure was standardized by assurance of comparable levels of the house-keeping gene GAPDH, amplified with PCR.

Three-dimensional structural analysis of rBD1: A structural model was generated for the rat BD via homology modeling. The target structure was predicted via alignment to a set of three human $\mathrm{BD}$ templates (isoforms 1,2 and 3 respectively) for which experimental structural characterization was available ${ }^{[19 ; 22]}$. The rat BD sequence was aligned to the human templates via the CLUSTAL-W program ${ }^{[23]}$, using the Blosum 30 substitution matrix, a gap-opening penalty of 10 and a gap-extension penalty of 0.1 . The resulting alignment and the corresponding three-dimensional human BD peptide structures were then processed via the Modeller program ${ }^{[24]}$ to yield a structural prediction for the rBD1 target. Modeller's default simulated annealing cycles were used for structural refinement. Analysis of the peptide secondary structure and surface characteristics was carried out on the resulting structure via SYBYL (SYBYL 6.9.2; The Tripos Associates, 2004, St. Louis, MO).

\section{RESULTS}

Expression of rBD-1 in laser-excised cardiomyocytes and H9c2 cells: Expression of rBD1 in laser-excised normal rat cardiomyocytes as well as fetal rat heart myoblasts ( $\mathrm{H} 9 \mathrm{c} 2)$ was confirmed by a single $318 \mathrm{bp}$ transcript identified by gel electrophoresis on the RTPCR products (Fig.1). The nucleotide sequencing results from the rBD-1 amplicon are shown in Fig. 2.

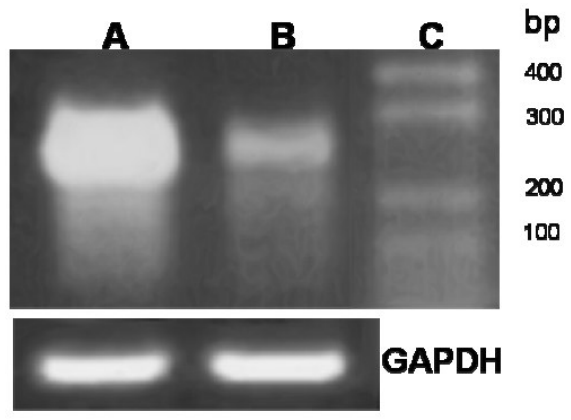

Fig.1: Tissue expression of rBD1 mRNA. $2 \mu \mathrm{g}$ of total RNA were subject to RT-PCR and $10 \mu \mathrm{l}$ of 40 -cycle aplicons were separated on $2 \%$ agarose gel and stained with ethidium bromide. RT-Expected products had a 318 bp in size. Laser-excised cardiomyocytes (line A), and fetal rat heart myoblasts; H9c2 (line B).

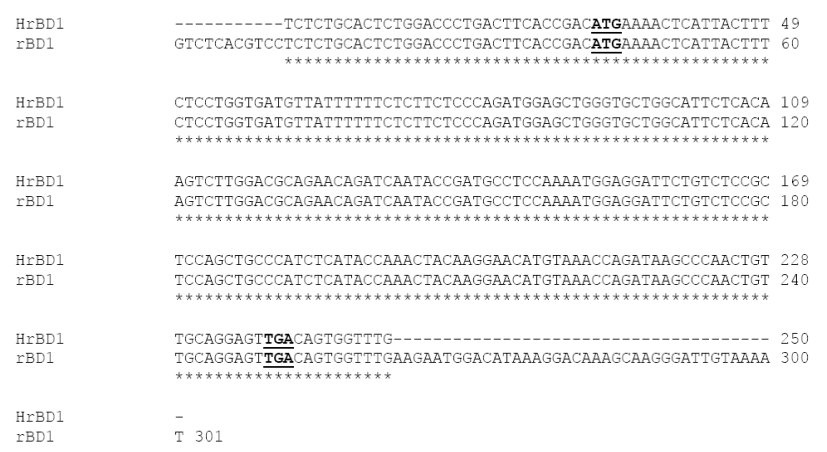

Fig.2: Full length cardiac rBD1 cDNA sequence showing the start and stop codons (bold and underlined). The cDNA sequence displayed a $99 \%$ homology with the BD previously described in rat kidney ${ }^{[15]}$

Comparison of heart rBD-1 with beta-defensins from other species: The open reading frame of the cardiac rBD cDNA was translated into a putative peptide with 69 amino acid residues: MKTHYFLLVMLFFLFSQM ELGAGILTSLGRRTDQYRCLQNGGFCLRSSCPSHT KLQGTCKPDKPNCCRS. Using CLUSTAL-W, the predicted amino acid sequence of the rat heart BD1 peptide was aligned to five BDs from other species. The cardiac rBD1 displayed the strongest identity with the renal rBD1 (99\%); mouse (88\%); human (53\%); gorilla (53\%); and Rhesus monkey (50\%) BDs (Fig.3).

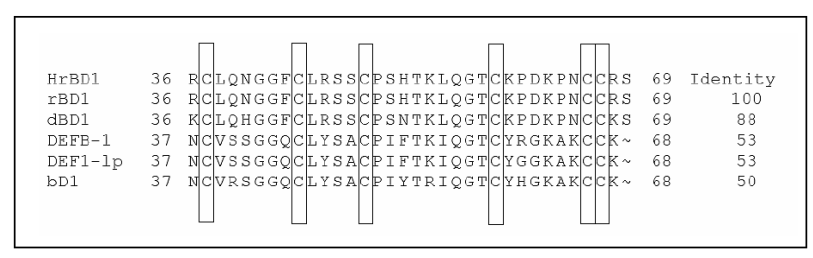

Fig.3: Amino acid alignment of the defensin-domain regions of the heart rat beta defensin (HrBD1) with other known beta-defensins. The conservative cysteine residues are framed. GenBank accession number: rBD1 (rat): AF093536, dBD1 (mouse): BC024380, DEF1 (Homo sapiens): BC047677, DEF1-like protein (gorilla): AAK61461, bD-1 (Macaca mulatta): AAK26258.

Three-dimensional structural analysis of rBD-1: The structure of rBD1 (Fig.4) was predicted through alignment with three NMR solution structures of human beta defensins 1-3 (PDB IDs: 1KJ5, 1FD4, and 1KJ6 respectively). The key six bridging cysteine motif was found to be conserved in the sequence alignment of rat 
and human defensins, and no alignment gaps were observed in the core region, thus suggesting similar three-dimensional packing in all cases. In terms of functionality, the rat structure was found to be much more similar to hBD1 (53\% identical; $86 \%$ homologous over the core 36 aligned residues) compared to either hBD2 (35\% identical; 59\% homologous over 37 core residues) or hBD3 (32\% identical; 59\% homologous over 37 residues). Within the core region, rBD1 and hBD1 displayed the same charge $(+4$ at $\mathrm{pH}=7.0)$ comparable to that of hBD2 (+5), but significantly less than that of hBD3 $(+8)$. rBD1 displayed a significantly greater number of amphoteric residues (6 histidines, asparagines and glutamines) than any of the human analogs (4 for hBD1, 2 each for hBD2 and hBD3). In place of these extra amphoteric residues were additional hydroxyl amino acids in the case of hBD1, nonpolar and hydrophobic species for hBD2 and bases for hBD3.
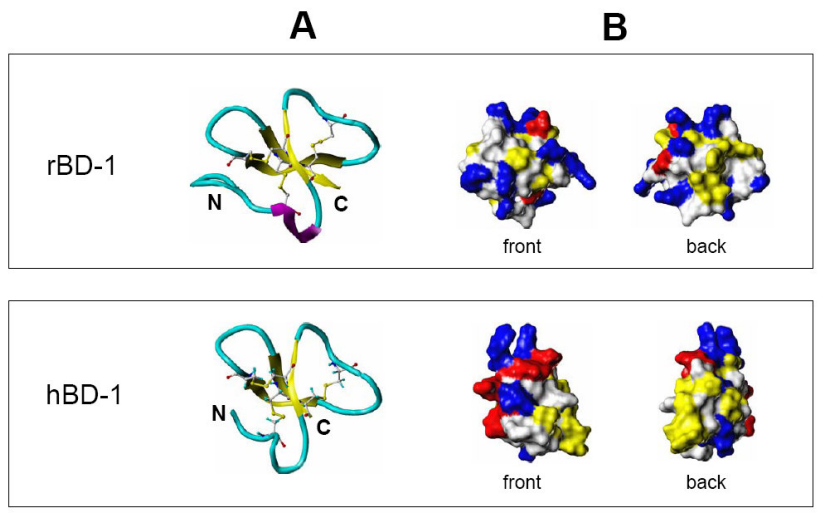

Fig.4: Secondary structure assignment and surface analysis for rBD1 and hBD1. (A) Ribbon diagrams showing alpha-helix (purple), betasheet (yellow), and coil (turquoise) secondary structure elements for the two peptides. (B) Front and back views of peptide solvent accessible surfaces. Acidic (anionic) residues are red, basic (cationic) residues are blue, and hydrophobic residues are yellow.

\section{DISCUSSION}

Innate immunity and inflammation have become central themes of investigations into a range of different disease processes, including common cardiovascular pathologies such as atherosclerosis and chronic heart failure ${ }^{[16]}$. Continual identification of specific effector molecules and immunological pathways potentially contributing to the development of such ailments is therefore indicated. The classical perception of the immune system as a complex but somewhat fixed entity of specialized organs, vessels and cells reacting in response to pathogens only has changed. A modernized view recognizes "danger" - more than foreignness - as the actual trigger mechanism of immunity, while also acknowledging the importance of non-immune cells and organs in host defense ${ }^{[9 ; 10]}$.

The heart possesses a germ-line encoded innate stress system, which is activated in response to different types of injury ${ }^{[6]}$. Recent studies have focused on the role of individual cytokines in development of cardiac disease and heart failure ${ }^{[7]}$. Attention has also been directed at identifying different toll-like receptors (TLRs) in the heart, and their potential implication in cardiovascular pathology ${ }^{[17]}$. TLRs are classical pattern recognition receptors utilizing NFKB-signaling - a shared pathway for induction of pro-inflammatory cytokines as well as BDs ${ }^{[3 ; 18]}$. BDs play a significant role in host defense, and some are up-regulated in disease ${ }^{[1 ; 3]}$. No studies have, however, focused on specifically identifying the expression pattern of heart BDs and their potential role in cardiac health and disease.

Our findings demonstrate the specific expression of a classically known epithelium-derived natural antibiotic peptide ( $\beta$-defensin) in non-epithelial cells (rat cardiomyocytes). By using the P.A.L.M. MicroBeam System, we were able to specifically excise clusters of cardiomyocytes in a non-contact, non-heatproducing fashion. This allowed us to perform gene expression studies on one specific cell type from the rat heart. The local expression of cardiac BDs may play a central role within an intrinsic innate immune response of the heart. Contextual studies are warranted to investigate the actual role of beta-defensins in cardiomyocytes further. It may be speculated that cardiac BDs act as a "second line of defense" within the framework of an innate immune response providing cardiomyocytes with a self-preserving mechanism when the immediate protecting barriers of the endothelial lining become disintegrated by damage. The presence of at least one $\mathrm{BD}$ isoform in cardiomyocytes further supports the notation that the heart is capable of producing innate effector molecules locally, as has previously been reported for certain cytokines.

Different BD isoforms have been identified within single organs ${ }^{[1]}$, and our preliminary data indicate that different $\mathrm{BD}$ isoforms are also present in the rat myocardium. Generally, the BD-1 isoform is known as a more ubiquitous and constitutively expressed isoform compared to other known BDs ${ }^{[19] .}$ The antimicrobial 
activity of this isoform is, however, potentially limited as it is not inducible by LPS ${ }^{[19-21]}$. It is possible that rBD1 act primarily as a local myocardial storage that can be mobilized and transformed into other BD isoforms with higher antimicrobial activities. A higher number of amphoteric sites in the rBD1 compared to hBDs would on the other hand indicate a strong $\mathrm{pH}$ functional dependence in rat relative to the human counterparts. Studies focused on tissues other than heart have shown that rBD1 is not directly up-regulated by the presence of certain bacterial components ${ }^{[20 ; 21]}$. It has furthermore been suggested, that over-expression of rBD1 may play a role in diabetic nephropathy in rats ${ }^{[15]}$. Based on the structural analysis of rBD1 it may, however, be hypothesized that the acid-base status in tissues may play a central role for the functional properties of this antimicrobial peptide. Alternatively, this isoform may possess other yet to be identified specific functional characteristics of potential significance in cardioprotection against non-infectious insults.

The present study is the first to not only identify a classical epithelial BD (rBD1) in cardiomyocytes from rat specifically isolated using position-ablation-lasermicrodissection, but also to demonstrate BD expression in embryonic cardiac tissue from rat. Investigation of $\mathrm{BD}$ expression in laser-excised cardiomyocytes is novel as it virtually eliminates the potential crosscontamination from other types of cells within the heart. Further investigations are warranted to identify the potential significance of cardiac BDs in heart disease.

\section{ACKNOWLEDGEMENTS}

This study was generously supported by COBRE/NIH grant P20 RR017686 (Dr. Melgarejo). The authors wish to thank Dr. Tere Ortega, Ms. Shannon Walsh and Mrs. Alicia Jackson for invaluable technical support.

\section{REFERENCES}

1. Ganz, T., 2005. Defensins and other antimicrobial peptides: a historical perspective and an update. Comb Chem High Throughput Screen, 8: 209-217.

2. Lehrer, R.I., 2004. Primate defensins. Nat Rev Microbiol, 2: 727-738.

3. Selsted, M.E. and A.J. Ouellette, 2005. Mammalian defensins in the antimicrobial immune response. Nat Immunol, 6: 551-557.

4. Zasloff, M., 2002. Antimicrobial peptides of multicellular organisms. Nature, 415: 389-395.
5. Doggrell, S.A., 2005. Recent advances in heart research. Drug News Perspect, 18: 58-72.

6. Wilson, E.M., A. Diwan, F.G. Spinale and D.L. Mann, 2004. Duality of innate stress responses in cardiac injury, repair, and remodeling. J Mol Cell Cardiol, 37: 801-811.

7. Diwan, A., T. Tran, A. Misra and D.L. Mann, 2003. Inflammatory mediators and the failing heart: a translational approach. Curr Mol Med, 3: 161-182.

8. Binder, C.J., M.K. Chang, P.X. Shaw and Y.I. Miller, 2002. Hartvigsen K, Dewan A, Witztum JL. Innate and acquired immunity in atherogenesis. Nat Med, 8: 1218-1226.

9. Feuerstein G.Z., P. Libby and D.L. Mann, 2003. Inflammation - A new frontier in cardiac disease and therapeutics. In: Inflammation and Cardiac Diseases (eds G.Z. Feuerstein, P. Libby and D.L. Mann) pp.1-5. Birkhauser Verlag, Basel, Switzerland.

10. Matzinger, P., 1994. Tolerance, danger, and the extended family. Annu Rev Immunol, 12: 9911045.

11. Morrison, G., F. Kilanowski, D. Davidson and J. Dorin, 2002. Characterization of the mouse beta defensin 1, Defb1, mutant mouse model. Infect Immun, 70: 3053-3060.

12. Davis, E.G., Y. Sang and F. Blecha, 2004. Equine beta-defensin-1: full-length cDNA sequence and tissue expression. Vet Immunol Immunopathol, 99: 127-132.

13. Zhang, G., H. Wu, J. Shi, T. Ganz, C.R. Ross and F. Blecha, 1998. Molecular cloning and tissue expression of porcine beta-defensin-1. FEBS Lett, 424: 37-40.

14. Jia, H.P., B.C. Schutte, A. Schudy, R. Linzmeier, J.M. Guthmiller, G.K. Johnson, B.F. Tack, J.P. Mitros, A. Rosenthal, T. Ganz and P.B. McCray Jr., 2001. Discovery of new human beta-defensins using a genomics-based approach. Gene, 263: 211218.

15. Page, R.A. and A.N. Malik, 2003. Elevated levels of beta defensin-1 mRNA in diabetic kidneys of GK rats. Biochem Biophys Res Commun, 310: 513-521.

16. Feuerstein, G.Z., P. Libby and D.L. Mann, 2003. Summary: Immune and inflammatory modulators as potential therapeutic targets for cardiac diseases. In: Inflammation and Cardiac Diseases (eds G.Z. Feuerstein, P. Libby and D.L. Mann) pp. 407-409. Birkhauser Verlag, Basel, Switzerland. 
17. Frantz, S., D. Fraccarollo, H. Wagner, T.M. Behr, P. Jung, C.E. Angermann, G. Ertl and J.Bauersachs, 2003. Sustained activation of nuclear factor kappa $\mathrm{B}$ and activator protein 1 in chronic heart failure. Cardiovasc Res, 57: 749-756.

18. Takeda, K. and S. Akira, 2004. TLR signaling pathways. Semin Immunol, 16: 3-9.

19. Schibli, D.J., H.N. Hunter, V. Aseyev, T.D. Starner, J.M. Wiencek, P.B. McCray Jr., B.F. Tack and H.J. Vogel, 2002. The solution structures of the human beta-defensins lead to a better understanding of the potent bactericidal activity of HBD3 against Staphylococcus aureus. J Biol Chem, 277: 82798289.

20. Palladino, M.A., T.A. Mallonga and M.S. Mishra, 2003. Messenger RNA (mRNA) expression for the antimicrobial peptides beta-defensin-1 and betadefensin- 2 in the male rat reproductive tract: betadefensin-1 mRNA in initial segment and caput epididymidis is regulated by androgens and not bacterial lipopolysaccharides. Biol Reprod, 68: 509-515.
21. Hiratsuka, T., M. Nakazato, Y. Date, H. Mukae and S. Matsukura, 2001. Nucleotide sequence and expression of rat beta-defensin-1: its significance in diabetic rodent models. Nephron, 88: 65-70.

22. Hoover, D.M., K.R. Rajashankar, R. Blumenthal, A. Puri, J.J. Oppenheim, O. Chertov and J. Lubkowski, 2000. The structure of human betadefensin-2 shows evidence of higher order oligomerization. J Biol Chem, 275: 32911-32918.

23. Thompson, J.D., D.G. Higgins and T.J. Gibson, 1994. CLUSTAL W: improving the sensitivity of progressive multiple sequence alignment through sequence weighting, position-specific gap penalties and weight matrix choice. Nucleic Acids Res, 22: 4673-4680.

24. Sanchez, R. and A. Sali, 2000. Comparative protein structure modeling. Introduction and practical examples with modeller. Methods Mol Biol, 143: 97-129. 